\title{
The Alcohol Use Disorders Identification Test (AUDIT): A review of graded severity algorithms and national adaptations
}

\author{
Thomas F. Babor ${ }^{1}$ and Katherine Robaina ${ }^{1}$ \\ ${ }^{1}$ University of Connecticut, School of Medicine, Farmington, Connecticut, United States
}

\begin{abstract}
Aims: Since it was first released in 1989, the Alcohol Use Disorders Identification Test (AUDIT) has generated a large amount of research to evaluate its psychometric properties. The purpose of this review is to critically evaluate the literature relevant to applications of the AUDIT in screening, brief intervention, and treatment referral programs, and identify national adaptations of the AUDIT to country-specific health, education, and reimbursement needs.

Methods: Methods comprised a search of the world literature published since 2004, combined with review articles published since 1997.

Findings: We identified 431 studies of the AUDIT, including 386 articles, 26 review papers, and 11 book chapters since 2004, with a six-fold increase in the last decade. The factor structure of the AUDIT items remains unclear, but the weight of evidence supports a two-factor model. Despite the translation of the AUDIT into numerous languages, the alcohol consumption questions were rarely adapted to suit cultural or national conditions. Although numerous studies have supported the recommended cutoff thresholds for a possible alcohol use disorder, only three studies evaluated the classification accuracy of the AUDIT's graded severity system.
\end{abstract}

Conclusions: Further development of the AUDIT score's severity zones is needed to guide intervention selection in clinical settings.

\section{Introduction}

In 1982, at the request of the World Health Organization (WHO), an international group of addiction scientists began to develop the Alcohol Use Disorders Identification Test (AUDIT). The aim of the test is to identify people with hazardous and harmful alcohol use (Babor, Higgins-Biddle, Saunders, \& Monteiro, 2001) in primary health care. The AUDIT is composed of 10 questions that represent three domains: alcohol consumption (Items 1-3), dependence
(Items 4-6), and alcohol-related consequences (Items 710). The total score ranges from 0 to 40 .

While the main purpose of the AUDIT is to identify persons with hazardous or harmful patterns of alcohol use, the test developers were also interested in using the continuum of its total score to assign patients to more intensive levels of intervention (see Table 1).

Table 1

AUDIT zones, scores, risk levels, and recommended interventions

\begin{tabular}{llll}
\hline $\begin{array}{c}\text { Total AUDIT } \\
\text { Score }\end{array}$ & \multicolumn{1}{c}{ Zone } & \multicolumn{1}{c}{ Risk level } & \multicolumn{1}{c}{ Intervention } \\
\hline $0-7$ & Zone I & Abstinence/ Lower-risk drinking & Feedback \\
$8-15$ & Zone II & Hazardous use & Brief intervention \\
$16-19$ & Zone III & Harmful use & Brief intervention, further monitoring, and diagnostic evaluation \\
$20-40$ & Zone IV & Possible dependence & Referral to specialist treatment \\
\hline
\end{tabular}

Adapted from Babor et al., 2001

Correspondence: Thomas Babor, PhD, MPH, University of Connecticut Health Center Department of Community Medicine, 263 Farmington Avenue, MC 6325, Farmington, CT 06030-6325. Telephone: 860.649.5459; E-mail: babor@uchc.edu

Financial support: The authors are grateful to JBS International for the support provided to conduct this literature review and for resources provided by SAMHSA/CSAT under Contract Number HHSS283200700003I/HHSS28300002T.

Keywords: Alcohol Use Disorders Identification Test, screening, AUDIT, alcohol use disorder, review, translations 
In the 2001 AUDIT manual (Babor et al., 2001, p. 28), research guidelines were provided to facilitate the continued development of the test and its applications to early intervention, secondary prevention, and referral to treatment. One suggestion was to investigate whether the AUDIT's questions could be scored as subscales to produce separate assessments of hazardous use, harmful use, and alcohol dependence (AD). If screening could be differentiated into these separate domains, perhaps the AUDIT could be more effectively linked to specific educational and treatment approaches to secondary prevention, with separate foci on hazardous use, harmful drinking, and AD. Alternatively, it was suggested that to the extent that the AUDIT total score provides a general measure of severity, it may be useful for stepped-care approaches to clinical management (i.e., providing the lowest level of intervention that addresses the patient's immediate needs), followed by the next higher intensity of care if the patient does not respond. Further research was also recommended to find the optimal cutoff points that are most appropriate for simple advice, brief counseling, and more intensive treatment. In another section of the manual (p. 32, "Translation and Adaptation to Specific Languages, Cultures and Standards"), it was suggested that "the drinking customs and beverage preferences of certain countries may require adaptation of questions to conform to local conditions.”

Since the publication of the first AUDIT Practitioner's Guide (Babor et al., 1989), the AUDIT has been validated in different settings and translated into many different languages. The AUDIT-C, a short version composed of the three consumption questions, has been found to produce results as valid and reliable as the full AUDIT (Bush et al., 1998; Frank et al., 2008), although poor performance of the first item has been reported in Argentina (Cremonte et al., 2010) and Germany (Rumpf et al., 2002). In addition to psychometric research comparing the AUDIT score to the results of diagnostic evaluations, the AUDIT has been used in numerous clinical and epidemiological investigations. Research has also been conducted to evaluate the graded scoring system that allows clinicians to estimate that extent of alcohol involvement and assign patients to appropriate levels of intervention.

Although research on the AUDIT has been the subject of several systematic reviews (Allen, Litten, Fertig, \& Babor, 1997; Meneses-Gaya, Zuardi, Loureiro, \& Crippa, 2009; Reinert \& Allen, 2002, 2007), including one on Chinese translations of the AUDIT (Li, Babor, Hao, \& Chen, 2011) and one meta-analysis (Berner, Kriston, Bentele, \& Härter, 2007), there have been no reviews of the AUDIT's graded scoring system and its ability to screen for Alcohol Use Disorders (AUDs) as defined by Diagnostic and Statistical Manual of Mental Disorders, Fourth and Fifth Editions (DSM-IV and DSM-5) and the International Classification of Diseases (ICD-10). In this article we review research on the use of the total AUDIT score as a severity measure to assign patients to appropriate levels of intervention ranging from brief intervention to referral to formal treatment for AD. Standard drink definitions, which inform low risk drinking guidelines, vary across countries. Therefore, we also searched for references to national adaptations that could be used to inform the further development of AUDIT screening programs.

\section{Methods}

A literature search that used Scopus database was conducted to identify studies published on the AUDIT for the past 10 years (2004 to present). Keywords used were "Alcohol Use Disorders Identification Test." Because the manual calling for national adaptations and the use of the graded scoring system had not been released until late 2002, we reasoned that it was unlikely for articles on these topics to be published before 2004. The search yielded 431 studies, including 386 articles, 26 review papers, 11 book chapters, and eight editorials/letters. Search results originated from 50 different countries, with most (40.6\%) from the United States.

All studies reporting psychometric properties (i.e., validation and reliability studies) were reviewed for their attention to the detection of AUDs and possible assignment of patients to recommended intervention levels based on severity scores. The growing body of research on the nonEnglish versions of the AUDIT was also included. Studies on the effectiveness of a brief intervention for managing hazardous drinking were not included, nor were book chapters and editorials/letters. We also consulted reviews of earlier studies of the AUDIT that have been summarized in several systematic reviews specific to the AUDIT (Allen et al., 1997; Li et al., 2011; Meneses-Gaya et al., 2009; Reinert \& Allen, 2002, 2007). In 1997, Allen et al. published the first narrative review of research on the AUDIT. As the AUDIT continued to generate a wideranging body of research, the review was updated again in 2002 and in 2007 (Reinert \& Allen, 2002, 2007). A review by Meneses-Gaya et al. (2009) highlighted research validating the AUDIT's applications beyond primary care, including other health care and community settings and non-English -speaking populations.

Combining the results of the systematic reviews with individual studies published since 2004, this review focuses on four types of research: 1) classification accuracy in the identification of AUDs; 2) research on the AUDIT severity zones; 3) studies of the AUDIT items' factor structure; and 4) translations and national adaptations.

\section{Results}

Figure 1 shows the number of articles published each year since 2004. The figure indicates a six-fold increase in the published literature, from approximately 10 articles in 2004 to over 80 in 2014. Combined with the studies included in the earlier systematic reviews, there have been more than 500 research reports and literature reviews of the AUDIT since its development. 
Figure 1

AUDIT publications, 2004-2014

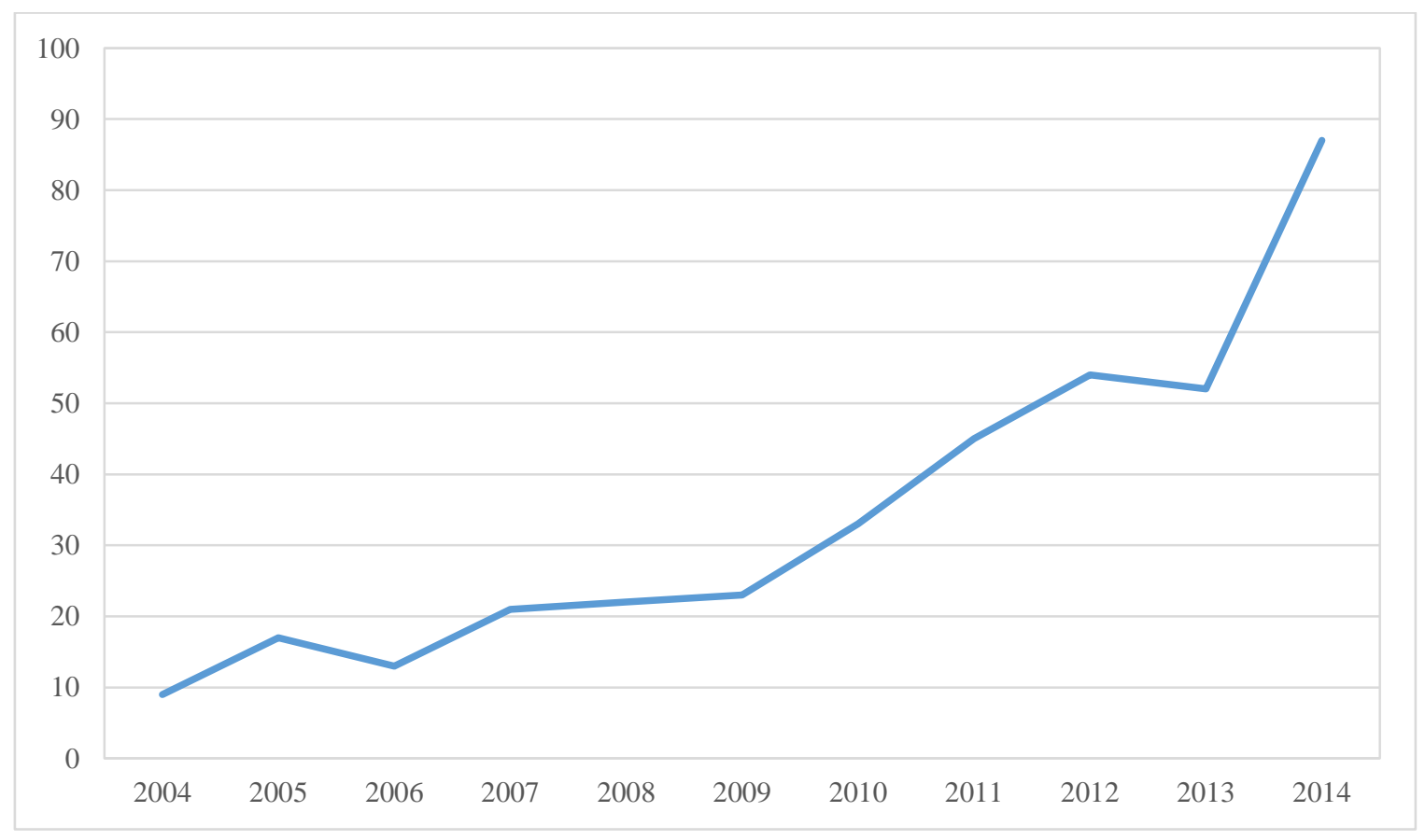

\section{Detection of Alcohol Use Disorders}

Several studies have examined the psychometric properties of the AUDIT and its capacity to correctly identify individuals with alcohol abuse or AD as defined by DSMIV. Moussas et al. (2009) assessed the validity of the Greek version in a population of 109 adults who met the criteria for $\mathrm{AD}$ as specified in the DSM-IV and 109 controls and found that a cut score of 8 detects $97 \%$ of the alcohol-dependent individuals and has a high sensitivity and specificity. Grache et al. (2005) examined the validity of the French AUDIT's capacity to diagnose alcohol abuse or AD and found that the AUDIT showed a good capacity to classify dependent patients with cut scores of $\geq 13$ for males and for females, and to classify hazardous drinkers with cut scores of $\geq 7$ for males and $\geq 6$ for females. Aalto, Alho, Halme, and Seppä (2009) recommend cut scores of $\geq 7$ for men and $\geq 5$ for women for adoption in Finland. These lower cutoff points for women are also supported by systematic reviews (Reinert \& Allen, 2002, 2007), which recommend a cut score of 5 or 6 for women. Lundin, Hallgren, Balliu, and Forsell (2015) found the optimal cutoff for $\mathrm{AD}$ was $\geq 6$ for both men and women in a sample of the general population in Sweden. A cut score of 5 was found to be most appropriate for identifying alcohol abuse in Malaysia (Yee, Adlan, Rashid, Habil, \& Kamali, 2015) and AUDs and/or at-risk consumption (with the exception of AD) in an outpatient population in Northern Germany (Dybek et al., 2006). In a validation study of a Nepali version of the AUDIT $(N=1,068)$, Pradhan et al. (2012) suggest a cutoff value of 11 for $\mathrm{AD}$ for both men and women. Authors recommend cutoff scores of $\geq 5$ for males and $\geq 4$ for females to identify hazardous drinking, based on the WHO's definition of a standard drink (10g ethanol).

Kim et al. (2014) assessed the validity of a Korean version of the AUDIT (AUDIT-KR), revised to reflect the decrease in alcohol by volume of soju, the most commonly consumed alcoholic beverage in Korea. Using a sample of 435 patients visiting a national university hospital for a comprehensive medical exam, the researchers found a cut score of 3 was appropriate to identify at-risk drinking in both men and women, while cut scores of 10 and 8 were appropriate for identifying AUDs for men and women, respectively.

Many studies have assessed the reliability of the AUDIT and its concurrent validity using the Composite International Diagnostic Interview (CIDI), version 2.1 (WHO, 1997), which enables diagnoses of alcohol use disorders according to both DSM-IV and ICD-10 criteria. Adapting the Spanish version of the AUDIT to Chilean language and culture, Alvarado et al. (2009) conducted a validation study among 93 primary care patients (60\% male) in Chile. Adjusting the standard drink size (13g versus $10 \mathrm{~g}$ in the international version), the authors found a cut score of 6 is effective for detecting hazardous drinking (sensitivity, 0.83; specificity, 0.88) and a cut score of 9 (sensitivity, 0.87; specificity, 0.85) for dependence. A study by Pérula et al. (2009) among primary care patients in Spain $(N=614)$ assessed the psychometric properties of the AUDIT and its capacity to correctly diagnose alcohol abuse or AD, as defined by DSM-IV, ICD-10, and hazardous drinking. Researchers found that a cut score of 7 points is effective for detecting hazardous drinking 
(sensitivity, 0.92; specificity, 0.92); and the optimum cut score is 6 to detect possible dependence. According to ICD-10 criteria, sensitivity was 0.82 , specificity 0.82 , while sensitivity was 0.88 and specificity 0.83 , according to DSM-IV. In assessing the validity of the AUDIT in a sample of Spanish female patients, Pérula et al. (2005) found the highest sensitivity and specificity values for the cutoff point of 6, according to DSM-IV and ICD-10 criteria.

Researchers in Australia examined the validity of the AusAUDIT using ICD-10 criteria for harmful use and dependence, and Australian National Health and Medical Research Council's (NH\&MRC) recommended drinking limits (no more than 40g per day for men and no more than $20 \mathrm{~g}$ per day for women). Authors found that cutoff scores of 11 for men and 9 for women were optimal for identifying those drinking above NH\&MRC recommended limits. The optimal cut scores for ICD harmful use and dependence were 10 and 17 respectively (Degenhardt et al., 2001).

Lima et al. (2005) validated the AUDIT against ICD-10 in a Brazilian sample of 166 middle-class HMO clients (59\% female). The receiver operating characteristic analysis showed that the optimal cutoff 7 (females) and 8 (males) ICD-10 diagnosis of AUDs. Giang et al. (2005) also found that the 7 and 8 cutoff points were effective for detecting harmful use (sensitivity, 1.00; specificity, 0.70) and dependence (sensitivity, 0.94; specificity, 0.87) using ICD10 criteria among men in rural Vietnam. In a Finnish study $(N=600)$ using a cut score of 8 , the AUDIT demonstrated good sensitivity (0.78) and specificity (0.86) to measure high-volume drinking and dependence (sensitivity, 0.79; specificity, 0.85) according to ICD criteria (Selin, 2006).

Six validation studies published in English on the Chinese version of the AUDIT conducted in Taiwan, Hong Kong (Chen, Chen, \& Cheng, 2004, 2005; Leung \& Arthur, 2000; Tsai, Tsai, Chen, \& Liu, 2005; Wu et al., 2008), and Tibet (Guo et al., 2008) were identified. Guo et al. (2008), adapting the measurement of a standard drink to account for Tibetans' self-brewed alcoholic beverages, found that cutoff values of 10 and 13 were effective for identifying alcohol abuse and $\mathrm{AD}$, respectively, with sensitivity and specificity for both $>0.84$. Tsai et al. (2005) found that a cut score of 11 had the best predictive value for $\mathrm{AD}$ (sensitivity, 0.94; specificity, 0.63), while a cut score of 8 (sensitivity, 0.96; specificity, 0.85) is recommended for identifying harmful drinking in a Taiwanese clinic $(N=112)$. Using the Mandarin Chinese version of the AUDIT, Wu and colleagues (2008) found that a cutoff of 7 was optimal to detect hazardous drinking among hospitalized Chinese patients in Taiwan.

Comparing the AUDIT to the CIDI to determine cutoff values for differentiating between hazardous drinking and AD among a sample of Sri Lankan married men, Silva, Jayawardana, and Pathmeswaran (2008) found that cut scores of 7 and 16 demonstrated the best sensitivity, specificity, and positive predictive values for hazardous drinking and $\mathrm{AD}$, respectively.
Among community outreach and de-addiction center samples in North India (Pal, Jena, \& Yadav, 2004) found the AUDIT optimal cutoff scores were higher than WHO recommendations: 16 (sensitivity, 0.85; specificity, 0.89) for harmful use and 24 (sensitivity, 0.69; specificity, 0.87) for AD, per ICD-10 criteria. Tuunanen, Aalto, and Seppä (2007) and von der Pahlen et al. (2008) also recommended higher cut scores for use in Finland.

\section{AUDIT Zones}

The WHO guidelines for the AUDIT specify four "zones" of scores that indicate increasing levels of alcohol-related risk, as shown in Table 1. However, validation data for these score ranges are limited. Our search located only three studies (Donovan, Kivlahan, Doyle, Longabaugh, \& Greenfield, 2006; Johnson, Lee, Vinson, \& Seale 2013; Rubinsky, Kivlahan, Volk, Maynard, \& Bradley, 2010) that reported on AUDIT zones.

Donovan et al. (2006) examined the concurrent validity of the AUDIT total score and intervention zones in a sample of mostly alcohol-dependent outpatient clients $(N=1,134)$. Indicators of severity of dependence and alcohol-related problems increased linearly with total score and differed significantly across AUDIT zones. The highest zone (20+) was markedly different with respect to severity from the other two zones. Individuals with scores of 20 or above had a greater severity of dependence; experienced more negative consequences related to their drinking; had higher levels of craving; experienced more symptoms associated with withdrawal; had more abnormal values on laboratory indicators of prolonged heavy drinking; and had a higher rate of prior treatment. Members of this group also endorsed an abstinence goal more strongly and had the highest score on a readiness-to-change measure. It was concluded that the AUDIT total score "appears to provide an index of severity of dependence in a sample of alcoholdependent individuals seeking out-patient treatment, extending its potential utility beyond its more traditional role as a screening instrument in general populations" (Donovan et al., 2006, p. 1696).

Using interview data on 625 primary care patients, Johnson et al. (2013) found that optimal AUDIT scores for detecting "unhealthy" alcohol use were 5 for men and 3 for womenscores lower than generally used. Authors also found that the WHO-recommended cutoff scores for zones III and IV revealed $100 \%$ specificity for both genders at cutoff values for both zones; however, the scores had low sensitivity in both men (zone III, 0.16 and zone IV, 0.06) and women (zone III, 0.12 and zone IV, 0.03). In using all possible cutoff values to assess dependence, the authors found the optimal cutoff points based on sensitivity and specificity to be $\geq 6$ for men (sensitivity, 0.84 ; specificity, 0.76 ) and $\geq 4$ for women (sensitivity, 0.88; specificity, 0.76), although the positive predictive value is low $(0.43$ and 0.28 respectively). Specificity can be increased, however, by adding a 30-day binge drinking measure.

A cross-sectional study among adult family medicine patients by Rubinsky et al. (2010) found that among men $(n=392)$, AUDIT score ranges of 5-10, 11-14, and 15-40 
were associated with post-screening probabilities of DSMIV past-year $\mathrm{AD}$ ranging from $18 \%$ to $87 \%$, as calculated by stratum-specific likelihood ratios. Among women $(n=927)$, AUDIT score ranges of 3-4, 5-8, 9-12, and 1340 were associated with post-screening probabilities of past-year $\mathrm{AD}$ ranging from $6 \%$ to $94 \%$. The authors identified the optimal scores for AUDIT zone IV at $\geq 15$ for men and $\geq 13$ for women.

Beyond the evidence that the AUDIT zones reflect increasing levels of alcohol involvement and treatment need, there is no clear consensus that the recommended ranges are optimal, in part because they have not been validated against criteria that would permit a standard of graded severity.

\section{Factor structure}

The AUDIT has been traditionally scored as a three-factor screening instrument: consumption (Items 1-3), dependence (Items 4-6), and related consequences (Items 7-10) (Rist, Glöckner-Rist, \& Demmel, 2009; Saunders, Aasland, Babor, de la Fuente, \& Grant, 1993; Tuliao, Landoy, \& McChargue, 2016). Several studies (El-Bassel, Schilling, Ivanoff, Chen, \& Hanson, 1998; Leung \& Arthur, 2000; Skipsey, Burleson, \& Kranzler, 1997) suggest that the AUDIT is measuring one factorhazardous drinking.

Most studies on the factor structure of the AUDIT, however, support a two-factor model, suggesting that the AUDIT is measuring patterns of intake/consumption (Items 1-3) and alcohol problems (Items 4-9) (Guo et al., 2008; Hallinan, Burleson, \& Kranzler, 2011; Hildebrand \& Noteborn, 2015; Lima et al., 2005; Shevlin \& Smith, 2007; Shields, Guttmannova, \& Caruso, 2004; Yee et al., 2015). Peng et al. (2012) examined the factor structure of the AUDIT as applied in general population surveys conducted in 15 countries $(N=27,478)$. A two-factor model representing consumption and dependence/consequences provided the best fit to the data. Findings from a crosssectional Finnish study including men and women across different age groups (von der Pahlen et al., 2008) ( $N=$ 9,131 ) and a large study based on data collected in two different multisite randomized controlled clinical trials (Doyle, Donovan, \& Kivlahan, 2007) also support a twofactor solution for both men and women.

It is possible that the two-factor findings may result in part from methods variance as the consumption items have similar response scales and are interdependent. A single factor implies graded severity of alcohol involvement that can be measured by means of a single score. Two factors imply that consumption questions are best used to detect hazardous drinking, with remaining questions scored to determine whether referral to specialized treatment is indicated (higher scores) or primary-care management (intermediate scores) is indicated.

\section{Translations and National Adaptations}

The AUDIT has been translated into Spanish, Slovak, Norwegian, French, German, Russian, Japanese, Swahili, Chinese, Czech, Vietnamese, Hindi, Korean, and several other languages. Our review of the literature indicates that only a few of these translations (Korean, Chinese, and Tibetan) have made any adaptations of the first three questions to take into account variations in standard drink sizes or national recommendations regarding moderate drinking levels. The Spanish version has been modified for use in Chile, and the English version was modified for use in Australia to take into account the recommended drinking limits (men 40g per day, women 20g/ day).

\section{Discussion}

Currently, the AUDIT (Saunders et al., 1993) is one of the most frequently used tools for screening for $\mathrm{AD}$ (Selin, 2006). It is also the alcohol screening test used most often in clinical and epidemiological research, as evidenced by its expanding applications and increasing number of publications (see Figure 1). Since the first scientific article on its development was published (Saunders et al., 1993), it has been cited 3,360 times, with citations steadily increasing each year and the most citations occurring in $2013(n=413)$.

The AUDIT was designed to screen for hazardous and harmful drinking, but validity studies have typically used as the criterion structured diagnostic interviews like CIDI, which do not measure hazardous drinking. While most studies looked at cut scores of 7 (females) and 8 (males) and above, only three examined the complete set of four AUDIT zones. Unlike screening instruments that focus on the presence of $\mathrm{AD}$ or alcoholism, an advantage of the AUDIT is that it is capable of identifying individuals along the full spectrum of alcohol misuse, providing an opportunity not only for brief interventions to reduce hazardous drinking but also referral to treatment at higher levels of severity.

As indicated in this and other reviews, most of the psychometric research with the AUDIT has focused on its screening capabilities in general population samples, primary care clinics, and hospital settings. Typically, a cut score of 7 (females) or 8 (males) is used to differentiate individuals without problems from those with potential problems (8-40) (Reinert \& Allen, 2007), with some consideration for lower scores among women and those over 65 (Babor et al., 2001). However, there is little information concerning the meaning or clinical usefulness of scores that fall in the problematic alcohol use range above this cut score.

Donovan et al. (2006) conducted one of the few studies on the AUDIT zones, concluding that the zones II and III are very similar on numerous indices of severity. They suggest that differentiating zone II from zone III may not be useful for clients with AUD. Because zone IV reflects meaningful differentiation from zones II and III, these authors argue that there may be further differentiation of severity possible within zone IV.

The AUDIT manual recommends that patients scoring in zone IV should be offered diagnostic evaluation and 
treatment in specialty settings. This group represents the most severely impaired drinkers who demonstrate numerous social, psychological, and medical problems. However, the range of AUDIT scores in zone IV may be too broad to differentiate adequately the most appropriate setting, treatment modality (e.g., pharmacotherapy or behavioral intervention), or intensity of treatment to be sought in a referral.

Further development and refinement within the AUDIT zones may be necessary to allow them to be more helpful in treatment selection in clinical settings. Future research should test empirically derived and gender-specific cutpoints for meaningful zones reflecting severity within treatment-seeking individuals with AUD. Hasin et al. (2013) have proposed the rationale for a dimensional index of severity of disorder, a suggestion that was incorporated into DSM-5, which allows a diagnostic classification of AUD and a rating of the severity. Severity is assessed by a count of the number of criteria endorsed and is classified into the broad dimensions of mild, moderate, and severe. This would seem to be a fruitful area for further research.

\section{References}

Aalto, M., Alho, H., Halme, J. T., \& Seppä, K. (2009). AUDIT and its abbreviated versions in detecting heavy and binge drinking in a general population survey. Drug and Alcohol Dependence, 103(1-2), 2529.

Allen, J. P., Litten, R. Z., Fertig, J. B., \& Babor, T. (1997). A review of research on the Alcohol Use Disorders Identification Test (AUDIT). Alcoholism: Clinical and Experimental Research, 21(4), 613-619.

Alvarado, M. E., Garmendia, M. L., Acuña, G., Santis, R., \& Arteaga, O. (2009). Validez y confiabilidad de la versión Chilena del Alcohol Use Disorders Identification Test (AUDIT). Revista médica de Chile, 137(11), 1463-1468.

Babor, T. F., de la Fuente, J. R., Saunders, J., Grant, M. (1989). AUDIT The Alcohol Use Disorders Identification Test: Guidelines for use in primary health care. WHO/MNH/DAT 89.4, World Health Organization, Geneva.

Babor, T. F., Higgins-Biddle, J., Saunders, J. B., \& Monteiro, M. G. (2001). AUDIT The Alcohol Use Disorders Identification Test: Guidelines for use in primary care (2nd ed.). Geneva, Switzerland: World Health Organization.

Berner, M. M., Kriston, L., Bentele, M., \& Härter, M. (2007). The Alcohol Use Disorders Identification Test for detecting at-risk drinking: A systematic review and meta-analysis. Journal of Studies on Alcohol and Drugs, 68(3), 461-473.

Bush, K., Kivlahan, D. R., McDonell, M. B., Fihn, S. D., \& Bradley, K. A. (1998). The AUDIT alcohol consumption questions (AUDIT-C): An effective brief screening test for problem drinking. Archives of Internal Medicine, 158(16), 1789-1795.

Chen, C. H., Chen, W. J., \& Cheng, A. T. (2004). A prevalence and identification of alcohol use disorders among non-psychiatric inpatients in one general hospital. General Hospital Psychiatry, 26, 219-225.

Chen, C. H., Chen, W. J., \& Cheng, A. T. (2005). New approach to the validity of the alcohol use disorders identification test: Stratum-specific likelihood ratios analysis. Alcoholism: Clinical and Experimental Research, 29, 602-608.

Cremonte, M., Ledesma, R. D., Cherpitel, C. J., \& Borges, G. (2010). Psychometric properties of alcohol screening tests in the emergency department in Argentina, Mexico and the United States. Addictive Behaviors, 35(9), 818-825.

Degenhardt, L. J., Conigrave, K. M., Wutzke, S. E., \& Saunders, J. B. (2001). The validity of an Australian modification of the AUDIT questionnaire. Drug and Alcohol Review, 20, 143-154.

Donovan, D. M., Kivlahan, D. R., Doyle, S. R., Longabaugh, R., \& Greenfield, S. F. (2006). Concurrent validity of the Alcohol Use Disorders Identification Test (AUDIT) and AUDIT zones in defining levels of severity among out-patients with alcohol dependence in the COMBINE study. Addiction, 101, 1696-1704.

Doyle, S. R., Donovan, D. M., \& Kivlahan, D. R. (2007). The factor structure of the Alcohol Use Disorders Identification Test (AUDIT). Journal of Studies on Alcohol and Drugs, 68, 474-479.

Dybek, I., Bischof, G., Grothues, J., Reinhardt, S., Meyer, C., Hapke, U., . . . Rumpf, H. J. (2006). The reliability and validity of the Alcohol Use Disorders Identification Test (AUDIT) in a German general practice population sample. Journal of Studies on Alcohol, 67, 473-481.

El-Bassel, N., Schilling, R., Ivanoff, A., Chen, D. -R., \& Hanson, M. (1998). Assessing the World Health Organization's Alcohol Use Disorder Identification Test among incarcerated women. Journal of Offender Rehabilitation, 26, 71-89.

Frank, D., DeBenedetti, A. F., Volk, R. J., Williams, E. C., Kivlahan, D. R., \& Bradley, K. A. (2008). Effectiveness of the AUDIT-C as a screening test for alcohol misuse in three race/ethnic groups. Journal of General Internal Medicine, 23, 781-787.

Gache, P., Michaud, P., Landry, U., Accietto, C., Arfaoui, S., Wenger, O., \& Daeppen, J. B. (2005). The Alcohol Use Disorders Identification Test (AUDIT) as a screening tool for excessive drinking in primary care: Reliability and validity of a French version. Alcoholism: Clinical and Experimental Research, 29(11), 2001-2007.

Giang, K. B., Spak, F., Dzung, T. V., \& Allebeck, P. (2005). The use of AUDIT to assess level of alcohol problems in rural Vietnam. Alcohol and Alcoholism, 40(6), 578-583.

Guo, W., Lanzi, G., Luobu, O., Ma, X., Zhen, P., Ji, Y., . . . Wang, Y. (2008). An epidemiological survey of alcohol use disorders in a Tibetan population. Psychiatry Research, 159(1), 56-66.

Hallinan, P., McGilloway, S., Dempster, M., \& Donnelly, M. (2011). Factor structure and validity of the Alcohol Use Disorders Identification Test (AUDIT) in a 
sample of mentally disordered offenders. Journal of Forensic Psychiatry and Psychology, 22(4), 586-602.

Hasin, D. S., Auriacombe, M., Borges, G., Bucholz, K., Budney, A., Crowley, T., . . . Wall, M. M. (2013). The DSM5 field trials and reliability of Alcohol Use Disorder. The American Journal of Psychiatry, 170(4), 442-443.

Hildebrand, M., \& Noteborn, M. G. C. (2015). Exploration of the (interrater) reliability and latent factor structure of the Alcohol Use Disorders Identification Test (AUDIT) and the Drug Use Disorders Identification Test (DUDIT) in a sample of Dutch probationers. Substance Use and Misuse, 50(10), 1294-1306.

Johnson, J. A., Lee, A., Vinson, D., \& Seale, J. P. (2013). Use of AUDIT-Based measures to identify unhealthy alcohol use and alcohol dependence in primary care: A validation study. Alcoholism: Clinical and Experimental Research, 37, E253-E259.

Kim, C. -G., Kim, J. S., Jung, J. -G., Kim, S. -S., Yoon, S. J., \& Suh, H. -S. (2014). Reliability and validity of Alcohol Use Disorder Identification Test-Korean Revised Version for screening at-risk drinking and alcohol use disorders. Korean Journal of Family Medicine, 35(1), 2-10.

Leung, S. F., \& Arthur, D. (2000). The alcohol use disorders identification test (AUDIT): Validation of an instrument for enhancing nursing practice in Hong Kong. International Journal of Nursing Studies, 37, 57-64.

Li, Q., Babor, T. F., Hao, W., \& Chen, X. (2011). The Chinese translations of Alcohol Use Disorders Identification Test (AUDIT) in China: A systematic review. Alcohol and Alcoholism, 46(4), 416-423.

Lima, C. T., Freire, A. C., Silva, A. P., Teixeira, R. M., Farrel, M., \& Farrel, M. (2005). Concurrent and construct validity of the AUDIT in urban Brazilian sample. Alcohol and Alcoholism, 40, 584-589.

Lundin, A., Hallgren, M., Balliu, N., \& Forsell, Y. (2015). The use of Alcohol Use Disorders Identification Test (AUDIT) in detecting alcohol use disorder and risk drinking in the general population: Validation of AUDIT using schedules for clinical assessment in neuropsychiatry. Alcoholism: Clinical and Experimental Research, 39, 158-165.

Meneses-Gaya, C., Zuardi, A. W., Loureiro, S. R., \& Crippa, J. A. (2009). Alcohol use disorders identification test (AUDIT): An updated systematic review of psychometric properties. Psychology and Neuroscience, 2(1), 83-97.

Moussas, G., Dadouti, G., Douzenis, A., Poulis, E., Tzelembis, A., Bratis, D., ... \& Lykouras, L. (2009). The alcohol use disorders identification test (AUDIT): Reliability and validity of the Greek version. Annals of General Psychiatry, 8(11), 1-5.

Pal, H. R., Jena, R., \& Yadav, D. (2004). Validation of the Alcohol Use Disorders Identification Test (AUDIT) in urban community outreach and de-addiction center samples in north India. Journal of Studies on Alcohol, 65, 794-800.

Peng, C. -Z., Wilsnack, R. W., Kristjanson, A. F., Benson, P., \& Wilsnack, S. C. (2012). Gender differences in the factor structure of the Alcohol Use Disorders
Identification Test in multinational general population surveys. Drug and Alcohol Dependence, 124(1-2), 5056. http://doi.org/10.1016/j.drugalcdep.2011.12.002

Pérula de Torres, L. A., Fernández-García, J. A., AriasVega, R., Muriel-Palomino, M., Márquez-Rebollo, E., \& Ruiz-Moral, R. (2005). Validity of AUDIT test for detection of disorders related with alcohol consumption in women. Medicina Clínica (Barcelona), 125, 727-730.

Pérula de Torres, L. A., Rebollo, E. M., Ruiz-Moral, R., Fernández-García, J. A., Vega, R. A., \& Palomino, M. M. (2009). Diagnostic usefulness of the Alcohol Use Disorders Identification Test (AUDIT) questionnaire for the detection of hazardous drinking and dependence on alcohol among Spanish patients. The European Journal of General Practice, 15(1), 15-21.

Pradhan, B., Chappuis, F., Baral, D., Karki, P., Rijal, S., Hadengue, A., \& Gache, P. (2012). The alcohol use disorders identification test (AUDIT): validation of a Nepali version for the detection of alcohol use disorders and hazardous drinking in medical settings. Substance Abuse Treatment, Prevention, and Policy, 7(1), 1.

Reinert, D. F., \& Allen, J. P. (2002). The Alcohol Use Disorders Identification Test (AUDIT): A review of recent research. Alcoholism: Clinical and Experimental Research, 26(2), 272-279.

Reinert, D. F., \& Allen, J. P. (2007). The Alcohol Use Disorders Identification Test: An update of research findings. Alcoholism: Clinical and Experimental Research, 31(2), 185-199.

Rist, F., Glöckner-Rist, A., \& Demmel, R. (2009). The Alcohol Use Disorders Identification Test revisited: Establishing its structure using nonlinear factor analysis and identifying subgroups of respondents using latent class factor analysis. Drug and Alcohol Dependence, 100(1-2), 71-82.

Rubinsky, A. D., Kivlahan, D. R., Volk, R. J., Maynard, C., \& Bradley, K. A. (2010). Estimating risk of alcohol dependence using alcohol screening scores. Drug and Alcohol Dependence, 108(1-2), 29-36.

Rumpf, H. J., Hapke, U., Meyer, C., \& John, U. (2002). Screening for alcohol use disorders and at-risk drinking in the general population: psychometric performance of three questionnaires. Alcohol and Alcoholism, 37(3), 261-268.

Saunders, J. B., Aasland, O. G., Babor, T. F., de la Fuente, J. R., \& Grant, M. (1993). Development of the Alcohol Use Disorders Identification Test (AUDIT): WHO collaborative project on early detection of persons with harmful alcohol consumption II. Addiction, 88(6), 791-804.

Selin, K. H. (2006). Alcohol Use Disorder Identification Test (AUDIT): What does it screen? Performance of the AUDIT against four different criteria in a Swedish population sample. Substance Use \& Misuse, 41(14), 1881-1899.

Shevlin, M, \& Smith, G. W. (2007). The factor structure and concurrent validity of the Alcohol Use Disorder Identification Test based on a nationally representative UK sample. Alcohol \& Alcoholism, 42, 582-587. 
Shields, A. L., Guttmannova, K., \& Caruso, J. C. (2004). An examination of the factor structure of the Alcohol Use Disorders Identification Test in two high-risk samples. Substance Use and Misuse, 39(7), 11611182.

Silva, P., Jayawardana, P., \& Pathmeswaran, A. (2008). Concurrent validity of the Alcohol Use Disorders Identification Test (AUDIT). Alcohol \& Alcoholism, 43, 49-50.

Skipsey, K., Burleson, J. A., \& Kranzler, H. R. (1997). Utility of the AUDIT for identification of hazardous or harmful drinking in drug-dependent patients. Drug and Alcohol Dependence, 45(3), 157-163.

Tsai, M. -C., Tsai, Y. -F., Chen, C. -Y., \& Liu, C. -Y. (2005). Alcohol Use Disorders Identification Test (AUDIT): Establishment of cut-off scores in a hospitalized Chinese population. Alcoholism: Clinical and Experimental Research, 29, 53-57.

Tuliao, A. P., Landoy, B. V. N., \& McChargue, D. E. (2016). Factor structure and invariance test of the alcohol use disorder identification test (AUDIT): Comparison and further validation in a U.S. and Philippines college student sample. Journal of Ethnicity in Substance Abuse, 15(2), 127-143. doi:10.1080/15332640.2015.1011731

Tuunanen, M., Aalto, M., \& Seppä, K. (2007). Binge drinking and its detection among middle-aged men using AUDIT, AUDIT-C and AUDIT-3. Drug and Alcohol Dependence, 26, 295-299.

von der Pahlen, B., Santtila, P., Witting, K., Varjonen, M., Jern, P., Johansson, A., \& Sandnabba, N. K. (2008). Factor structure of the Alcohol Use Disorders Identification Test (AUDIT) for men and women in different age groups. Journal of Studies on Alcohol and Drugs, 69, 616-621.

Wu, S. I., Huang, H. C., Liu, S. I., Huang, C. R., Sun, F. J., Chang, T. Y., ... . Jeng, K. S. (2008). Validation and comparison of alcohol-screening instruments for identifying hazardous drinking in hospitalized patients in Taiwan. Alcohol \& Alcoholism, 43, 577-582.

World Health Organization. (1997) Composite International Diagnostic Interview. Version 2.1. Geneva, Switzerland: World Health Organization.

Yee, A., Adlan, A. S. A., Rashid, R. R., Habil, H., \& Kamali, K. (2015). Validation of the Alcohol Use Disorders Identification Test (AUDIT)-Bahasa Malaysia version among a group of alcohol users. Journal of Substance Use, 20(4), 229-233. 